\title{
ON DEFICIENCY GRADIENT OF GROUPS
}

\author{
ADITI KAR AND NIKOLAY NIKOLOV
}

\begin{abstract}
Deficiency gradient is a higher dimensional analogue of rank gradient. In this paper, we give a combinatorial proof that the fundamental group of a simply connected complex of amenable groups has deficiency gradient zero. We apply this to establish the vanishing of deficiency gradient in special linear groups over polynomial rings and number fields, and in Artin groups for which the nerve of the Coxeter matrix is simply connected. This implies that the first and second $l^{2}$ Betti numbers vanish for these Artin groups without recourse to the $K(\pi, 1)$ conjecture. We propose a conjecture about the stabilisation of deficiency gradient, which characterises groups with 2-dimensional classifying spaces.
\end{abstract}

\section{INTRODUCTION}

Throughout the paper, the phrase a (normal) chain in a group $G$ will refer to a descending sequence $G>H_{1}>H_{2}>H_{3}>\ldots$ of finite index (normal) subgroups in $G$ such that the intersection $\cap_{i} H_{i}$ is the residual $\mathfrak{R}(G)$ of $G$. The residual of a group is the intersection of all its subgroups of finite index and is trivial in any group that is residually finite.

Let $G$ be a finitely presented group. Set $d(G)$ to be the cardinality of a minimal generating set and $\delta(G)$ to be the deficiency of $G$. More precisely,

$$
\delta(G)=\max \{|S|-|R| \mid G:=\langle S \mid R\rangle\}
$$

We denote by $b_{n}(G)=\operatorname{dim}_{\mathbb{Q}} H_{n}(G, \mathbb{Q})=b_{n}(X)$, where $X=K(G, 1)$ is a classifying space for $G$ (i.e. a connected $C W$-complex $X$ with contractible universal cover and fundamental group $G)$, and $b_{n}(X)$ denotes the $n$-th Betti number of $X$. Note that $\delta(G) \leq b_{1}(G) \leq d(G)$. Therefore for a finite index subgroup $H \leq G$,

$$
\frac{\delta(H)-1}{[G: H]} \leq \frac{d(H)-1}{[G: H]} \leq d(G)-1
$$

Starting with the presentation $\langle S \mid R\rangle$ for $G$ one obtains a Schreier presentation for $H$ with $[G: H](|S|-1)+1$ generators and $[G: H]|R|$ relations showing that

$$
\frac{\delta(H)-1}{[G: H]} \geq \delta(G)-1 .
$$

It is well-known that $\delta(G) \leq b_{1}(G)-b_{2}(G)$ as a special case of the Morse inequalities $[13, \S 4$, p. 210]. 
Let $G$ be a finitely presented group and let $\left\{H_{i}\right\}$ be a normal chain in $G$. The sequence $\left\{\frac{\delta\left(H_{i}\right)-1}{\left[G: H_{i}\right]}\right\}$ is non-decreasing and bounded above by $d(G)-1$ and so we make the following definition.

Definition 1. The deficiency gradient of $G$ with respect to $\left(H_{i}\right)$ is

$$
\Delta\left(G,\left(H_{i}\right)\right)=\lim _{i \rightarrow \infty} \frac{\delta\left(H_{i}\right)-1}{\left[G: H_{i}\right]} .
$$

This notion has received some attention recently. In [1], deficiency gradient is studied using analytic tools while in [8], the authors study asymptotic growth of homology groups of residually free groups. In this note we investigate deficiency gradient with the tools of combinatorial group theory.

Recall that the limit $R G\left(G,\left(H_{i}\right)\right)=\lim _{i \rightarrow \infty} \frac{d\left(H_{i}\right)-1}{\left[G: H_{i}\right]}$ is called the rank gradient [4] of $G$ with respect to the chain $\left(H_{i}\right)$. Trivially one has $\Delta\left(G,\left(H_{i}\right)\right) \leq$ $R G\left(G,\left(H_{i}\right)\right)$.

Let $\beta_{i}(G)$ denote the $i$-th $l^{2}$-Betti number of the group $G$. Then Proposition 4 below gives

$$
\beta_{1}(G)-\beta_{2}(G) \geq \Delta\left(G,\left(H_{i}\right)\right)
$$

for any chain $\left(H_{i}\right)$ in $G$. If the chain $\left(H_{i}\right)$ is normal with trivial intersection we could ask by analogy with the similar question for rank gradient whether equality always holds above. Finding any examples where the inequality is strict seems to be a hard problem.

1.1. Groups with two dimensional classifying spaces. The deficiency gradient is easy to compute for groups $G$ which have finite two-dimensional classifying space $K(G, 1)$. Examples of such groups are surface groups or more generally, torsion-free one relator groups and, direct products of two free groups.

Lemma 2. If a group $G$ has a finite two-dimensional classifying space $K(G, 1)$, then $\delta(G)=1-\chi(G)$ and consequently, $\delta(H)-1=[G: H](\delta(G)-$ 1) for every subgroup $H$ of finite index in $G$.

Consequently, $\Delta\left(F_{n} \times F_{m},\left(H_{i}\right)\right)=-(n-1)(m-1)$ for any chain $\left(H_{i}\right)$ while the deficiency gradient of a torsion-free one relator group defined on $d$ generators is $d-2$, a number not surprisingly equal to the difference in its first and second $l^{2}$-Betti numbers.

We conjecture that the converse of Lemma 2 holds:

Conjecture 3. Let $G$ be a torsion-free residually finite finitely presented group such that $\delta(H)-1=[G: H](\delta(G)-1)$ for every subgroup $H$ of finite index in $G$. Then $G$ has a finite 2-dimensional space $K(G, 1)$.

Conjecture 3 is much in the character of open questions like the EilenbergGanea conjecture and the Relation gap problem in topological group theory. The following basic characterisation of groups with two dimensional classifying spaces may be useful: 
Lemma 4. Let $G$ be an infinite finitely presented group. Then $\delta(G)-1 \leq$ $\beta_{1}(G)-\beta_{2}(G)$ with equality if and only if $G$ has a two dimensional classifying space.

In particular any counterexample to Conjecture 3 will be a group $G$ with a normal chain $\left(H_{i}\right)$ such that $\Delta\left(G,\left(H_{i}\right)\right)<\beta_{1}(G)-\beta_{2}(G)$.

1.2. Economical groups: basic examples. If the deficiency gradient of an infinite group $G$ with respect to any chain is positive then $G$ has a finite index subgroup $H$ whose deficiency is at least 2. By a result of Baumslag and Pride [5] one deduces that $H$ and hence $G$ is large. Therefore deficiency gradient for amenable groups and torsion groups is at most 0 . In fact, deficiency gradient is almost always zero for amenable groups and they satisfy the stronger condition of being economical:

Definition 5. Let $\left(H_{i}\right)$ be a chain of finite index subgroups in an infinite finitely presented group $\Gamma$. We say that the chain $\left(H_{i}\right)$ is economical if each $H_{i}$ has a presentation of $d_{i}$ generators and $r_{i}$ relations such that

$$
\lim _{i \rightarrow \infty} \frac{d_{i}}{\left[\Gamma: H_{i}\right]}=\lim _{i \rightarrow \infty} \frac{r_{i}}{\left[\Gamma: H_{i}\right]}=0 .
$$

The group $\Gamma$ is economical if every normal chain in $\Gamma$ which intersects in $\mathfrak{R}(\Gamma)$ is economical. Note that this implies that $[\Gamma: \mathfrak{R}(\Gamma)]=\infty$.

If $\Gamma$ is economical then since $\delta\left(H_{i}\right) \geq d_{i}-r_{i}$ and the ratios $d_{i} / n_{i}$ and $r_{i} / n_{i}$ tend to zero we have $\Delta\left(\Gamma,\left(H_{i}\right)\right) \geq 0$. Now $0 \leq \Delta\left(\Gamma,\left(H_{i}\right)\right) \leq R G\left(\Gamma,\left(H_{i}\right)\right)=$ 0 and so both the deficiency gradient and the rank gradient of $\Gamma$ are zero. The slow groups defined in [8] are examples of economical groups.

Theorem 6. Let $G$ be a finitely presented, residually finite amenable group which is infinite. Then $G$ is economical.

Another class of examples of economical groups comes from the presence of normal subgroups which are also economical.

Proposition 7. Let $G$ be a finitely presented group which has a normal finitely presented infinite subgroup $N$. Let $\left(H_{i}\right)$ be a normal chain of $G$ such that the chain $\left(N \cap H_{i}\right)$ is economical. Then the chain $\left(H_{i}\right)$ is also economical. In particular, if a residually finite, finitely presented group $G$ has a normal economical subgroup, then $G$ itself is economical.

Our main result is a vanishing theorem for deficiency gradient of complexes of economical groups.

1.3. Complexes of groups. For definition and detailed properties of complexes of groups we refer to [7, Chapter III.C]. Here we summarise the properties that we need to state and prove Theorem 8 .

A scwol $P$ is a pair $(V, E)$ of two sets called vertices and edges together with maps $i, t: E \rightarrow V$. For an edge $a \in E$ we refer to $i(a)$ as the origin and to $t(a)$ as the terminus of $a$. A pair of edges $a, b \in E$ are called composable 
if $i(a)=t(b)$. In that case there is a third edge $c \in E$, called the product of $a$ and $b$ and denoted by $c=a b$ with the property that $i(c)=i(b)$ and $t(c)=t(a)$. The operation of taking products of edges is associative and in addition no edge $a$ is allowed to have $i(a)=t(a)$. An example which covers all applications in this paper is the case when the scwol $P$ is defined by a poset $(V,<)$. The vertex set of $P$ is $V$ and $E=\{(\sigma, \tau) \in V \times V \mid \sigma<\tau\}$. For $a=(\sigma, \tau) \in E$ we define $i(a)=\tau, t(a)=\sigma$.

A complex of groups $C$ over a scwol $P$ is a quadruple

$$
C=\left(P,\left(G_{\sigma}\right),\left(f_{a}\right),\left(g_{a, b}\right)\right)
$$

consisting of the following

- a scwol $P=(V, E)$,

- a family of groups $G_{\sigma}$ indexed by the elements $\sigma \in V$,

- group monomorphisms $f_{a}: G_{i(a)} \rightarrow G_{t(a)}$ for every edge $a \in E$,

- elements $g_{a, b} \in G_{i(a)}$ for every pair of composable edges $a, b \in E$.

The elements $g_{a, b}$ have to satisfy certain compatibility conditions described in [7, Definition III.C.2.1] (which play no role in our argument).

Every scwol $P$ has a realization $|P|$ as a polyhedral complex with edges $E$. We assume that $|P|$ is connected and choose a spanning tree $T$ of the edges $E$ of $P$. The fundamental group $\tilde{G}(C)$ is defined to be group generated by $E \cup\left(\cup_{\sigma \in V} G_{\sigma}\right)$ subject to the following four families of relations R1-R4:

R1: All relations of the groups $G_{\sigma}, \sigma \in V$,

R2: Relations $a b=g_{a, b} c$ for composable pairs of edges $a, b \in E$ with product $c=a b$.

R3: $f_{a}(x)=a x a^{-1}$ for all $a \in E$ and $x \in G_{i(a)}$,

R4: $a=1$ for all edges $a$ in $T$.

A complex $C$ is called simple if all elements $g_{a, b}$ are identity. A complex $C$ is developable if the inclusion homomorphism $G_{\sigma} \rightarrow \tilde{G}(C)$ is injective for each $\sigma \in V$. In that case we will identify the group $G_{\sigma}$ with its image in $\tilde{G}(C)$.

In the special case when $C$ is a simple complex whose scwol $P$ has a simply connected realization $|P|$ the additional identities $a=1$ hold in $\tilde{G}(C)$ for all edges $a \in E$. Therefore in this case the fundamental group $\tilde{G}(C)$ is just the direct limit (amalgam) of the system $\left(\left(G_{\sigma}\right),\left(f_{a}\right)\right)$.

In later application we will only need to consider simple complexes with simply connected realizations. The last condition is often easier to check when we consider from the start a simply connected simplicial compex $L$, together with a family of groups $G_{\sigma}$ for every simplex $\sigma \subset L$ and homomorphisms $f_{\sigma, \tau}: G_{\tau} \rightarrow G_{\sigma}$ for every pair $\sigma \subset \tau$. The swol $P=P_{L}$ is defined by the poset $(V,<)$ of simplices of $L$ ordered by inclusion and the geometric realization $|P|$ is the barycentric subdivision of $L$. Since $L$ is simply connected, so is $|P|$ and hence the fundamental group of the simple complex of 
groups $\left(P,\left(G_{\sigma}\right),\left(f_{\sigma, \tau}\right)\right)$ is the direct limit of the system $\left(\left(G_{\sigma}\right),\left(f_{\sigma, \tau}\right)\right)$. When there is no ambiquity we will write $\tilde{G}(L)$ for $\tilde{G}\left(P_{L},\left(G_{\sigma}\right),\left(f_{\sigma, \tau}\right)\right)$.

Our main result states that the fundamental group of a complex of economical groups is also economical:

Theorem 8. Let $C$ be a developable complex of groups over a scwol $P$ such that $|P|$ is connected. Let $\left(H_{i}\right)$ be a normal chain in $\Gamma:=\tilde{G}(C)$ such that for every vertex $\sigma \in V$ the chain $\left\{H_{i} \cap G_{\sigma}\right\}$ is economical in $G_{\sigma}$. Then $\left(H_{i}\right)$ is an economical chain of $\Gamma$ and $\Delta\left(\Gamma,\left(H_{i}\right)\right)=0$. In particular, if each $G_{\sigma}$ is economical and $G_{\sigma} \cap \mathfrak{R}(\Gamma)=\mathfrak{R}\left(G_{\sigma}\right)$ for each $\sigma \in L$, then $\Gamma$ is economical.

We note that in general it is hard to check whether a complex of groups is developable. However every complex $C$ gives rise to a developable complex on replacing the vertex groups $G_{\sigma}$ with their images in $\tilde{G}(C)$. Therefore if the groups $G_{\sigma}$ have the property that all their images are economical (e.g. if $G_{\sigma}$ are polycyclic) then the conclusion of Theorem 8 holds without assuming the developability of $C$.

1.4. Applications of Theorem 8. We can use Theorem 8 to show that many special linear groups and Artin groups are economical.

1.4.1. Steinberg groups and special linear groups. A unital ring $R$ is finitely presented if $R$ is isomorphic to $\mathbb{Z}\langle X\rangle / J$ where $\mathbb{Z}\langle X\rangle$ denotes the free associative ring on a finite set $X$, and $J$ is a finitely generated ideal of $\mathbb{Z}\langle X\rangle$.

Recall that the Steinberg group $S t_{n}(R)$ over a unital $\operatorname{ring} R$ is defined to be the group presented by generators $\left\{x_{r}(a) \mid r \in \Sigma, a \in R\right\}$ where $\Sigma$ is the root system of $S L_{n}$ of type $A_{n-1}$, subject to the following relations (cf. $[20], \S 5)$.

$$
\begin{array}{ll}
x_{r}(a) x_{r}(b)=x_{r}(a+b) & \\
{\left[x_{r}(a), x_{v}(b)\right]=1} & \text { if } r+v \notin \Sigma \cup\{0\} \\
{\left[x_{r}(a), x_{v}(b)\right]=x_{r+v}(a b)} & \text { if } r+v \in \Sigma
\end{array}
$$

for all $r, v \in \Sigma$ and all $a, b \in R$.

These relations imply in particular that for any $r \in \Sigma$ the set $X_{r}=$ $\left\{x_{r}(a) \mid a \in R\right\}$ is a subgroup of $S t_{n}(R)$ isomorphic to $(R,+)$

Theorem 9. Let $R$ be a finitely presented ring such that $(R,+)$ is torsion free. Assume that $n \geq 4$ and let $\left(H_{i}\right)$ be a normal chain in $S t_{n}(R)$ such that $X_{r} \cap \cap_{i=1}^{\infty} H_{i}=\{1\}$. Then the chain $\left(H_{i}\right)$ is economical. If in addition $R$ is a residually finite ring it follows that $S t_{n}(R)$ is economical.

In the case when $R$ is a polynomial ring the knowledge of the Milnor $K$-group $K_{2}(R)$ allows us to deduce that $S L_{n}(R)$ is economical when $n$ is sufficiently large. 
Corollary 10. Let $k \geq 0$ and let $R=\mathbb{Z}\left[x_{1}, \ldots, x_{k}\right]$ be the polynomial ring in $k$ variables. Let $n \geq k+4$. The group $S L_{n}(R)$ is economical and in particular has deficiency gradient zero with respect to any normal chain with trivial intersection.

When $k=0$ i.e. $R=\mathbb{Z}$ the above result has been obtained earlier in [1] using measure theoretic methods.

It will be interesting to find out if the group $S L_{3}(\mathbb{Z})$ is economical. In this connection we note that lattices in some semisimple Lie groups of rank 1 or 2 have strictly negative deficiency gradient, see [19], Theorem 3.

We can apply Theorem 8 to $S L_{3}$ over many rings of integers in number fields. In particular, the following Proposition shows that for every integer $m>1$ the group $S L_{3}\left(\mathbb{Z}\left[\frac{1}{m}\right]\right)$ is economical.

Proposition 11. Let $k$ be a number field and for a finite set $S$ of valuations of $k$ including all archimedean ones, let $R$ be the ring of $S$-integers of $k$. Assume that the group of units $R^{*}$ is infinite. Then $S L_{3}(R)$ is economical.

1.4.2. Artin groups. In [15] we showed that any Artin group $A$ with connected graph has rank gradient and $\beta_{1}(A)$ equal to zero. Let $L$ be the nerve of the Coxeter matrix of $A$. Assuming the $K(\pi, 1)$ conjecture for Artin groups it is proved in [10] that $\beta_{2}(A)=b_{1}(L)$. One may expect that $A$ has deficiency gradient zero whenever $b_{1}(L)=0$. We confirm this when $L$ is simply connected.

Theorem 12. Let $A$ be an Artin group such that the nerve $L$ of its associated Coxeter matrix is connected and simply connected. Then $A$ is economical.

When $A$ as above is right-angled, its deficiency gradient has been shown to be zero in [1].

Note that Theorem 12 and (1) imply the following, without recourse to the verity of the $K(\pi, 1)$ conjecture for Artin groups.

Corollary 13. Let $A$ be an Artin group such that the nerve $L$ of its associated Coxeter matrix is connected and simply connected. Then $\beta_{1}(A)=$ $\beta_{2}(A)=0$.

1.5. Lattices in $P S L(2, \mathbb{C})$. A well known open question in this subject asks whether the rank gradient vanishes with respect to any normal chain with trivial intersection for lattices in $P S L(2, \mathbb{C})$, c.f.[3]. From recent developments in 3-manifold theory due to Agol, Wise, et al (see [2] and references therein), we know that lattices in $\operatorname{PSL}(2, \mathbb{C})$ are virtually fibered. The noncocompact lattices in $P S L(2, \mathbb{C})$ are virtually free-by-cyclic i.e. some finite index subgroup is isomorphic to $F_{n} \rtimes \mathbb{Z}$. The co-compact lattices are virtually $\pi_{1}\left(\Sigma_{g}\right) \rtimes \mathbb{Z}$, where $\Sigma_{g}$ denotes a surface of genus $g \geq 2$. The groups $F_{n} \rtimes \mathbb{Z}$ are 2-dimensional and we can invoke Lemma 2 to conclude that with respect to any chain, the deficiency gradient of $F_{n} \rtimes \mathbb{Z}$ is zero. In the co-compact case, the $l^{2}$-cohomology is known to be trivial and so the deficiency gradient is at most zero. On the other hand, a cocompact lattice 
in $\operatorname{PSL}(2, \mathbb{C})$ comes with a well-known balanced presentation, arising from the Heegaard decomposition of the hyperbolic 3-manifold. This implies that the deficiency gradient of such a group with respect to any normal chain is at least zero. We conclude then that the deficiency gradient is always zero for both co-compact and non-cocompact lattices in $P S L(2, \mathbb{C})$. One may reformulate the vanishing of the rank gradient conjecture for these groups and ask whether lattices in $\operatorname{PSL}(2, \mathbb{C})$ are economical.

1.6. Free Products. It is well-known that the $l^{2}$-Betti numbers and rank gradient minus 1 are additive under free products. One might wonder if the same is true for deficiency gradient.

Question 14. Let $\Gamma_{1}$ and $\Gamma_{2}$ be two (torsion-free) residually finite groups and let $\left(H_{i}\right)$ be a normal chain in $\Gamma=\Gamma_{1} * \Gamma_{2}$. Is it always true that

$$
\Delta\left(\Gamma,\left(H_{i}\right)\right)=\Delta\left(\Gamma_{1},\left(H_{i} \cap \Gamma_{1}\right)\right)+\Delta\left(\Gamma_{2},\left(H_{i} \cap \Gamma_{2}\right)\right)+1 ?
$$

Unfortunately deficiency of groups is not additive under free products: [12] provides examples, e.g. $\left(C_{2} \times C_{2}\right) *\left(C_{3} \times C_{3}\right)$. The naive approach uses the Bass Serre tree $T$ for the free product. Each $H_{i}$ is a free product of several copies of $\Gamma_{1} \cap H_{i}$ and $\Gamma_{2} \cap H_{i}$ and possibly a free group which arises as the fundamental group of the graph $H_{i} \backslash T$. Therefore, starting with a presentation for $\Gamma_{1} * \Gamma_{2}$, we can easily write a presentation for each $H_{i}$. As this may not be the optimal presentation, we may conclude only that the following inequality holds:

$$
\Delta\left(\Gamma,\left(H_{i}\right)\right) \geq \Delta\left(\Gamma_{1},\left(H_{i} \cap \Gamma_{1}\right)\right)+\Delta\left(\Gamma_{2},\left(H_{i} \cap \Gamma_{2}\right)\right)+1 .
$$

Using the inequality (1) we can deduce a positive answer to Question 14 in a special case:

Proposition 15. Suppose that $\Gamma_{j}$ has deficiency gradient equal to $\beta_{1}\left(\Gamma_{j}\right)-$ $\beta_{2}\left(\Gamma_{j}\right)$ for any normal chain in $\Gamma_{j}(j=1,2)$. Then the same holds for $\Gamma_{1} * \Gamma_{2}$.

The rest of the paper is organised as follows. Lemmas 2 and 4, Theorem 6 and Proposition 7 are proved in Section 2. Our main theorem (Theorem 8 ) is proved in Section 3. The results 10 and 11 on special linear groups are proved in Section 4. Artin groups are discussed and Theorem 12 is proved in Section 5.

\section{Proofs.}

Proof of Lemma 2. Let $K$ be the universal cover of the classifying space $X=K(G, 1)$. Thus $K$ is a contractible 2-dimensional complex on which $G$ acts freely with compact quotient $X=K / G$. Let $e_{i}$ be the number of $i$-dimensional simplices of $X$. The Euler characteristic of $X$ is $\chi(X)=$ $e_{0}-e_{1}+e_{2}$ while $G=\pi_{1}(X)$ has a presentation with $e_{1}-e_{0}+1$ generators and $e_{2}$ relations. We have $b_{i}(G)=b_{i}(X)$ for $i=1,2$ and $b_{0}(X)=1$ since 
$X$ is connected. In addition $\delta(G) \leq b_{1}(G)-b_{2}(G)$, see for example [13], $\S 4$. Thus

$$
e_{1}-e_{0}-e_{2} \leq \delta(G)-1 \leq b_{1}(X)-1-b_{2}(X)=-\chi(X)=e_{1}-e_{0}-e_{2}
$$

and we deduce that $\delta(G)-1=-\chi(X)$. Similarly we find that $\delta(H)-1=$ $-\chi(K / H)$ and the lemma follows from $\chi(K / H)=[G: H] \chi(X)$.

Proof of Lemma 4. If $G$ has two dimensional classifying space then by Lemma 2 we have $\delta(G)-1=-\chi(G)=-\beta_{0}(G)+\beta_{1}(G)-\beta_{2}(G)$ and the result is clear since $\beta_{0}(G)=0$.

We now prove the converse. Assume that $G$ does not have 2-dimensional classifying space and let us choose a presentation $P=(S, R)$ for $G$ with $d$ generators and $r$ relations, such that $d-r=\delta(G)$. Let $X_{0}$ denote the 2-complex defined by the Cayley graph of $G$ where we add discs for every relation in $P$ at every vertex of $X_{0}$. By assumpition $X_{0}$ is not aspherical and therefore $H_{2}\left(X_{0}\right) \neq\{0\}$. Succesively adding cells in dimension 3 and higher we reach a complex $X$ which is homotopy equivalent to the classifying space $K(G, 1)$ and whose 2-skeleton is $X_{0}$. Our group $G$ acts freely on $X$ with $1, d, r$ orbits in dimensions $0,1,2$ respectively. Therefore we obtain a free resolution of $\mathbb{R} G$ modules

$$
\mathbb{R} \stackrel{\gamma_{0}}{\longleftarrow} \mathbb{R} G \stackrel{\gamma_{1}}{\longleftarrow}(\mathbb{R} G)^{d} \stackrel{\gamma_{2}}{\longleftarrow}(\mathbb{R} G)^{r} \stackrel{\gamma_{3}}{\longleftarrow}(\mathbb{R} G)^{k} \ldots
$$

such that the image of $\gamma_{3}$ is non-zero. This resolution induces a complex of $l^{2} G$ modules

$$
0 \stackrel{\tilde{\gamma}_{0}}{\longleftarrow} l^{2} G \stackrel{\tilde{\gamma}_{1}}{\longleftarrow}\left(l^{2} G\right)^{d} \stackrel{\tilde{\gamma}_{2}}{\longleftarrow}\left(l^{2} G\right)^{r} \stackrel{\tilde{\gamma}_{3}}{\longleftarrow}\left(l^{2} G\right)^{k} \longleftarrow \ldots
$$

where each map $\tilde{\gamma}_{i}$ is induced by the map $\gamma_{i}$. Denoting by $K_{i}=\operatorname{ker} \tilde{\gamma}_{i}$ and $J_{i}=\overline{\operatorname{Im} \tilde{\gamma}_{i}}$ we have $\beta_{i}(G)=\operatorname{dim}_{\mathcal{N}} K_{i} / J_{i+1}$ where for a $l^{2} G$ module $M$ we denote by $\operatorname{dim}_{\mathcal{N}} M$ its von Neumann dimension. Since $G$ is infinite we have $\beta_{0}(G)=0$. The rank-nullity theorem for von Neumann dimension gives that

$$
1-d+r-\operatorname{dim}_{\mathcal{N}} J_{3}=\beta_{0}(G)-\beta_{1}(G)+\beta_{2}(G)
$$

and since $\gamma_{3}$ is not zero we have $J_{3} \neq\{0\}$ and therefore $\operatorname{dim}_{\mathcal{N}} J_{3}>0$. The inequality follows.

Proof of Theorem 6. We use the following result from [23] (see also [4] for an alternative proof):

Theorem 16. Let $S$ be a finite generating set for an amenable group $G$ and let $\left(H_{i}\right)$ be an infinite normal chain in $G$ with trivial intersection. For any $\epsilon>0$ there is some $i \in \mathbb{N}$ and a transversal $T_{i}$ for $H_{i}$ in $G$ such that

$$
\left|\left\{(t, s) \in T_{i} \times S \mid t s \notin T_{i}\right\}\right|<\epsilon\left|T_{i}\right| .
$$

Now consider a presentation $\langle X, R\rangle$ for an amenable group $G$ and let $S$ be the image of the generating set $X$ in $G$. Let $m$ be the sum of the lengths of all relations in $R$ as words in $X$. For any $\epsilon>0$ let $T_{i}$ be a transversal for $H_{i}$ in $G$ as in the conclusion of the above theorem. For an element $a \in G$ write 
$\bar{a}$ for the unique element of $T_{i}$ such that $a \in \bar{a} H_{i}$. Then $H_{i}$ has the so-called Schreier presentation $\left\langle Y_{i}, W_{i}\right\rangle$ where $Y_{i}=\left\{e(t, s) \mid t \in T_{i}, s \in S\right\}$ and $W_{i}$ is a collection of $\left[G: H_{i}\right]|R|$ words in the generating set $Y_{i}$ with the property that any $e(t, s) \in Y_{i}$ occurs in at most $m$ of the relators $W_{i}$. Moreover the image of $e(t, s) \in Y_{i}$ in $H_{i}$ is the element $t s(\overline{t s})^{-1}$. Let $Y_{i}^{1}$ be the set of elements from $Y_{i}$ whose image in $H_{i}$ is non-trivial and let $W_{i}^{1}$ be the image of the relations from $W_{i}$ under the homomorphism $F\left\langle Y_{i}\right\rangle \rightarrow F\left\langle Y_{i}^{1}\right\rangle$ which sends the generators from $Y_{i} \backslash Y_{i}^{1}$ to identity.

We claim that $H_{i}=\left\langle Y_{i}, W_{i}\right\rangle$ has presentation $\left\langle Y_{i}^{1}, W_{i}^{1}\right\rangle$. Let $H_{i}^{1}=$ $\left\langle Y_{i}^{1}, W_{i}^{1}\right\rangle$ and consider the surjective homomorphism $f: H_{i} \rightarrow H_{i}^{1}$ obtained by setting all $Y_{i} \backslash Y_{i}^{1}$ to 1 . At the same time since $H_{i}$ is generated by the images of $Y_{i}^{1}$ and satisfies the relations $W_{i}^{1}$ there is a surjective homomorphism $h$ from $H_{i}^{1}$ to $H_{i}$ sending $Y_{i}^{1}$ to $Y_{i}^{1} \subseteq Y_{i}$. Since $f \circ h: H_{i}^{1} \rightarrow H_{i}^{1}$ is the identity we see that $h$ is injective and hence an isomorphism, proving the claim.

Now observe that $\left|Y_{i}^{1}\right|<\epsilon\left[G: H_{i}\right]$ by the choice of $T_{i}$. At the same time at most $\left|Y_{i}^{1}\right| m$ of the relations from $W_{i}^{1}$ are non-trivial as words in $F\left\langle Y_{i}^{1}\right\rangle$ because each element of $Y_{i}^{1}$ can appear in at most $m$ relations from $W_{i}^{1}$. Hence $H_{i}$ has presentation with at most $\epsilon\left[G: H_{i}\right]$ generators and at most $m \epsilon\left[G: H_{i}\right]$ relations. Since $m$ depends only on the initial presentation of $G$ while $\epsilon>0$ could be arbitrary small we deduce that the chain $\left(H_{i}\right)$ is economical.

Proof of Proposition 7. Let $G, N,\left(H_{i}\right)$ be as in the statement of the Proposition. As $N$ is finitely generated and $G$ is finitely presented, the quotient $Q=G / N$ is also finitely presented. We will show that each $H_{i}$ has a finite presentation with $d_{i}$ generators and $r_{i}$ relators such that $\lim _{i \rightarrow \infty} \frac{d_{i}}{\left[G: H_{i}\right]}=\frac{r_{i}}{\left[G: H_{i}\right]}=0$.

For each $i$, set $N_{i}=N \cap H_{i}$. Then the group $H_{i}$ is an extension of $N_{i}$ by $Q_{i} \cong \frac{H_{i} N}{N}$. Let $x_{i}=\left[N: N_{i}\right]$ and $y_{i}=\left[G: N H_{i}\right]$, note that $\lim _{i \rightarrow \infty} x_{i}=\infty$ while $\left[G: H_{i}\right]=x_{i} y_{i}$. The chain $\left(N_{i}\right)$ is economical in $N$ and each $N_{i}$ has a presentation $\left\langle X_{i} ; R_{i}\right\rangle$ with $a_{i}$ generators and $\rho_{i}$ relators such that $\lim _{i \rightarrow \infty} \frac{a_{i}}{x_{i}}=\lim _{i \rightarrow \infty} \frac{\rho_{i}}{x_{i}}=0$. Let $Q$ be presented by $k$ generators and $l$ relations. Each $Q_{i}$ has finite index $y_{i}$ in $Q$ and so we choose a finite Schreier presentation $\left\langle Y_{i} ; S_{i}\right\rangle$ for each $Q_{i}$ with $b_{i}=\left|Y_{i}\right|=y_{i}(k-1)+1$ generators and $\sigma_{i}=\left|S_{i}\right|=y_{i} l$ relators. Observe that $b_{i} \leq k y_{i}$ and $\sigma_{i}=l y_{i}$ for all $i$.

The group $H_{i}$ has a presentation with generating set $X_{i} \cup Y_{i}$. The relators are chosen to be $R_{i} \cup S_{i} \cup P_{i}$ where $P_{i}=\left\{s r s^{-1}=n_{r, s} \mid r \in X_{i}, s \in Y_{i}\right\}$. Therefore, we can take $d_{i}=a_{i}+b_{i}$ and $r_{i}=\rho_{i}+\sigma_{i}+a_{i} b_{i}$. It follows that

$$
\frac{d_{i}}{\left[G: H_{i}\right]}=\frac{a_{i}+b_{i}}{x_{i} y_{i}} \leq \frac{a_{i}}{x_{i}}+\frac{k}{x_{i}} ; \frac{r_{i}}{\left[G: H_{i}\right]}=\frac{\rho_{i}+\sigma_{i}+a_{i} b_{i}}{x_{i} y_{i}} \leq \frac{\rho_{i}}{x_{i}}+\frac{l}{x_{i}}+\frac{k a_{i}}{x_{i}} .
$$

Using that $\rho_{i} / x_{i}$ and $a_{i} / x_{i}$ both tend to zero with $i$, we conclude that $\lim _{i \rightarrow \infty} \frac{d_{i}}{\left[G: H_{i}\right]}=\lim _{i \rightarrow \infty} \frac{r_{i}}{\left[G: H_{i}\right]}=0$ and the chain $\left(H_{i}\right)$ is economical, as required. 


\section{Proof of Theorem 8}

Proof. Since $\Gamma$ is generated by economical groups with infinite intersections we have that $R G\left(\Gamma,\left(H_{i}\right)\right)=0$ by [15]. Therefore $\Delta\left(\Gamma,\left(H_{i}\right)\right) \leq 0$. It remains to show that $\Delta\left(\Gamma,\left(H_{i}\right)\right) \geq 0$ for which we need to exhibit a suitable presentation for each subgroup $H_{i}<\Gamma$. By Theorem III.C.3.13 and Corollary III.C.3.15 of [7] there is a universal development scwol $D=\left(V_{D}, E_{D}\right)$ on which $\Gamma$ act so that $P=\Gamma \backslash D$ and $C$ is the complex of groups associated to this action (as described in Definition III.C.2.9 (1) of [7]).

Let $Y_{j}=\left(V_{j}, E_{j}\right)$ be the scwol $H_{j} \backslash D$ and let $\mathcal{Y}_{j}$ be the complex of groups associated to the action of $H_{j}$ on $D$ which we describe below. By Corollary III.C.3.15 of [7] $H_{j} \simeq \tilde{G}\left(\mathcal{Y}_{j}\right)$.

Let $p_{j}: D \rightarrow Y_{j}$ be the natural projection morphism. Following Definition III.C.2.9 (1) of [7] we now describe the complex $\mathcal{Y}_{j}=\left(Y_{j}, H_{j, \sigma}, \psi_{j, a}, g_{j, a, b}\right)$.

For each $\sigma \in V_{j}$ choose a vertex $\bar{\sigma} \in V_{D}$ such that $p_{j}(\bar{\sigma})=\sigma$. For every edge $a \in E_{j}$ of $Y_{j}$ there is a unique edge $\bar{a} \in E_{D}$ such that $p_{j}(\bar{a})=a$ and $i(\bar{a})=\overline{i(a)}$. Choose $h_{j, a} \in \Gamma$ such that $h_{j, a} t(\bar{a})=\overline{t(a)}$. Let $H_{j, \sigma}=\operatorname{Stab}_{H_{i}}(\bar{\sigma})$ and define $\psi_{j, a}: H_{j, i(a)} \rightarrow H_{j, t(a)}$ be the injective homomorphism

$$
\psi_{j, a}(g)=h_{j, a} g h_{j, a}^{-1} \quad \forall g \in H_{j, i(a)}
$$

For pairs $a, b$ of composable edges of $E_{i}$ define

$$
g_{j, a, b}=h_{j, a} h_{j, b} h_{j, c}^{-1}
$$

where $c=a b$ is the product ot $a$ and $b$ in $E_{j}$.

The complex of groups $\mathcal{Y}_{j}$ is the quadruple $\left(Y_{j},\left(H_{j, \sigma}\right),\left(\psi_{j, a}\right),\left(g_{j, a, b}\right)\right)$.

Choose a spanning tree $T_{j}$ on the edge set $E_{j}$ of $Y_{j}$. Then $H_{j}$ is isomorphic to the fundamental group $\tilde{G}\left(\mathcal{Y}_{j}\right)$ of $\mathcal{Y}_{j}$ defined as follows:

Generators of $H_{j}$ are $\left\{H_{j, \sigma} \mid \sigma \in Y_{j}\right\} \cup E_{j}$. The relations of $H_{j}$ are of the following four types:

R1. All relations in the groups $H_{j, \sigma}$,

R2. $a b=g_{j, a, b} c$ for all composable pairs of edges $a, b \in E_{j}$ with $c=a b$.

R3. $\psi_{j, a}(x)=a x a^{-1}$ for all $x \in H_{j, i(a)}$ and all $a \in E_{j}$.

R4. $a=1$ for all $a \in T_{j}$.

We are now ready to prove that $\lim _{j \rightarrow \infty} \frac{\delta\left(H_{j}\right)-1}{\left[\Gamma: H_{j}\right]} \geq 0$. Consider the presentation of $H_{j}$ above. Let $n_{j}=\left[\Gamma: H_{j}\right]$. Denote by $\pi$ the morphism of scwols $Y_{j}=H_{j} \backslash D \rightarrow \Gamma \backslash D=P$. Recall that $V$ and $E$ denote the vertex and edge sets of $P$. For any vertex $\sigma \in V$ of $P$ choose a vertex $\sigma_{0} \in V_{j}$ of $Y_{j}$ such that $\sigma_{0} \in \pi^{-1}(\sigma)$ and moreover choose $\bar{\sigma} \in V_{D}$ to be equal to $\bar{\sigma}_{0}$. Now $\left|\pi^{-1}(\sigma)\right|=\left[\Gamma: H_{j} G_{\sigma}\right]=n_{j} / l_{j, \sigma}$ where $l_{j, \sigma}=\left[G_{\sigma}: H_{j, \sigma_{0}}\right]$. Moreover since $H_{j}$ is normal in $\Gamma$ we have that the isomorphism class of $H_{j, \sigma_{0}}=\operatorname{Stab}_{H_{j}}\left(\overline{\sigma_{0}}\right)=H_{j} \cap G_{\sigma}$ does not depend on the choice of $\sigma_{0} \in \pi^{-1}(\sigma)$. 
Since each $H_{j} \cap G_{\sigma}=H_{j, \sigma_{0}}$ is an economical chain in $G_{\sigma}$ we can choose a presentation $\left\langle X_{j, \sigma} \mid R_{j, \sigma}\right\rangle$ for the group $H_{j, \sigma_{0}} \leq G_{\sigma}$ such that

$$
\frac{\left|X_{j, \sigma}\right|}{l_{j, \sigma}} \rightarrow 0, \quad \frac{\left|R_{j, \sigma}\right|}{l_{j, \sigma}} \rightarrow 0 \text { and } l_{j, \sigma} \rightarrow \infty \text { as } j \rightarrow \infty .
$$

Now $H_{j}$ is generated by

$$
d_{j}:=\sum_{\sigma \in V}\left|X_{j, \sigma}\right| \frac{n_{j}}{l_{j, \sigma}}+\left|E_{j}\right|
$$

elements. The number $\left|E_{j}\right|$ of edges $a \in E_{j}$ of $Y_{i}$ is at most $\left|V_{j}\right||E|$ : There are at most $\left|V_{j}\right|$ choices for the initial vertex $x=i(a)$ of $a$ in $Y_{j}$ and then at most $|E|$ choices for $a$ because for any $x \in V_{j}$ the morphism $\pi$ induces a bijection between $\left\{a \in E_{j} \mid i(a)=x\right\}$ and $\{a \in E \mid i(a)=\pi(x)\}$. Now

$$
\left|V_{j}\right|=\sum_{\sigma \in V}\left|\pi^{-1}(\sigma)\right|=\sum_{\sigma \in V} \frac{n_{j}}{l_{j, \sigma}}
$$

which together with $l_{j, \sigma} \rightarrow \infty$ gives $\left|Y_{j}\right| / n_{j} \rightarrow 0$ with $i \rightarrow \infty$. Therefore $d_{j} / n_{j} \rightarrow \infty$. We now have to count the relations of $H_{j}$.

The number of relations in R1 is $r_{j, 1}:=\sum_{\sigma \in V}\left|R_{j, \sigma}\right| n_{j} / l_{j, \sigma}$ and so $r_{j, 1} / n_{j} \rightarrow$ 0 as $j \rightarrow \infty$.

The number $r_{j, 2}$ of relations in $\mathrm{R} 2$ is the number of composable pairs $a, b \in E_{j}$. There are $\left|Y_{j}\right|$ choices for $i(b)$, then at most $|E|$ choices for $b$ which determines $t(b)=i(a)$ and then at most $|E|$ choices for $a$. Therefore $r_{j, 2} \leq\left|Y_{j}\right||E|^{2}$ and again $r_{j, 2} / n_{j} \rightarrow 0$ with $j$.

The number $r_{j, 3}$ of relations in R3 is at most

$$
\sum_{a \in E_{j}}\left|X_{j, i(a)}\right| \leq \sum_{\tau \in V}|E| \frac{n_{j}}{l_{j, \tau}}\left|X_{j, \tau}\right|
$$

which shows that again $r_{j, 3} / n_{j} \rightarrow 0$ with $j \rightarrow \infty$.

Finally $\left|T_{j}\right| \leq\left|E_{j}\right| \leq|P|\left|Y_{j}\right|$ which shows that the number of relations R4 also grows sub-linearly in $n_{j}$.

This completes the proof that $\left(H_{j}\right)$ is economical and Theorem 8 follows.

\section{Special Linear Groups}

Proof of Theorem 9. Let $\Sigma$ be the root system $A_{n-1}$ with Weyl group $\operatorname{Sym}(n)$. The root system $\Sigma$ has the realization

$$
\Sigma=\left\{e_{i}-e_{j} \mid 1 \leq i, j \leq n, i \neq j\right\}
$$

where $e_{1}, \ldots, e_{n}$ is the standard orthonormal basis ot $\mathbb{R}^{n}$. Let $\mathcal{S}$ be the set of proper non-zero subspaces of $\mathbb{R}^{n}$ whose basis is a subset of $\left\{e_{1}, \ldots, e_{n}\right\}$. The Coxeter complex $\mathfrak{C}$ of $\operatorname{Sym}(n)$ is the simplicial complex with vertices $\mathcal{S}$ and simplices the collection $F$ all flags from $\mathcal{S}$ :

$$
F=\left\{\sigma=\left\{V_{1}, V_{2}, \ldots, V_{k}\right\} \mid V_{1} \subset V_{2} \subset \cdots \subset V_{k}, V_{j} \in \mathcal{S}, 1 \leq j \leq k\right\} .
$$


The set of maximal flags of $F$ is denoted by $F_{\max }$. These correspond to permutations of $\left\{e_{1}, \ldots, e_{n}\right\}$ and define the simplices of maximal dimension $n-2$ of $\mathfrak{C}$. The Coxeter complex $\mathfrak{C}$ of $\operatorname{Sym}(n)$ is a triangulation of the sphere $S^{n-2}$ and therefore $\mathfrak{C}$ is simply connected when $n \geq 4$.

Let $(F,<)$ be the poset of the flags in $F$ ordered by reverse inclusion, i.e. for flags $\sigma, \tau \in F$ we declare $\sigma<\tau$ whenever $\tau \subset \sigma$. The geometric realization $|F|$ of $F$ is the derived complex (barycentric subdivision) of $\mathfrak{C}$.

For a flag $\sigma=\left\{V_{1}, V_{2}, \ldots, V_{k}\right\} \in F$ let $r(\sigma)$ be the set of roots

$$
s(\sigma)=\left\{e_{i}-e_{j} \mid e_{i} \in V_{l}, e_{j} \notin V_{l} \text { for some } l \in\{1, \ldots, k\}\right\}
$$

Clearly if $\tau \subset \sigma$ then $s(\tau) \subset s(\sigma)$. If $\sigma \in F_{\max }$ is a maximal flag then $s(\sigma)$ is a set of positive roots $\left\{e_{\pi(a)}-e_{\pi(b)} \mid 1 \leq a<b \leq n\right\}$ with respect to some permutation $e_{\pi(1)}, \ldots, e_{\pi(n)}$ of $e_{1}, \ldots, e_{n}$. More generally the roots $s(\sigma)$ correspond to those elementary matrices in $S L_{n}$ which are strictly upper triangular with respect to the flag $\sigma$.

Let $Y$ be a finite generating set of the ring $R$ over $\mathbb{Z}$ such that $1 \in Y$.

For a flag $\sigma \in F$ let $G_{\sigma}$ be the subgroup of $S t_{n}(R)$ generated by $\left\{x_{r}(a) \mid r \in\right.$ $s(\sigma), a \in R\}$. It is well known that when $\sigma \in F_{\max }$ then $G_{\sigma}$ is isomorphic to the group $U_{n}(R)$ of upper uni-triangular $n \times n$ matrices with entries in $R$. The difficulty in applying Theorem 8 directly is that $G_{\sigma}$ is not finitely generated. We will resolve this by exhibiting $G_{\sigma}$ (and consequently $S t_{n}(R)$ ) as a direct limit of finitely presented groups which eventually stabilizes because $S t_{n}(R)$ is finitely presented.

For an integer $m \in \mathbb{N}$ let $Y_{m}=Y \cdot Y \cdots Y \subset R$ be the set of all products of some $m$ elements of $Y$ and note that $Y_{m} \subseteq Y_{m+1}$ since $1 \in Y$.

For $\sigma \in F$ define $G_{\sigma, m}$ to be the subgroup of $S t_{n}(R)$ generated by $\left\{x_{r}(a) \mid r \in s(\sigma), a \in Y_{m}\right\}$ and note that $G_{\sigma, m}$ is a finitely generated nilpotent group. If $\tau \subset \sigma$ are two flags in $F$ then $s(\tau) \subset s(\sigma)$ and therefore $G_{\tau, m}$ is contained in $G_{\sigma, m}$.

Choose and fix a natural number $m$ for the moment. Now define a simple complex of groups

$$
\mathcal{C}_{m}=\left(\mathcal{F},\left(G_{\sigma, m}\right)_{\sigma \in F},\left(f_{a, m}\right)_{a \in E}\right)
$$

over the scwol $\mathcal{F}=(F, E)$ of the poset $(F,<)$. Recall that the edge set $E$ of $\mathcal{F}$ is $E=\{(\sigma, \tau) \mid \sigma<\tau, \sigma, \tau \in F\}$.

For an edge $a=(\sigma, \tau) \in E$ of $\mathcal{F}$ let $f_{a, m}: G_{\tau, m} \rightarrow G_{\sigma, m}$ be the inclusion map.

Now define $\Gamma_{m}=\tilde{G}\left(\mathcal{C}_{m}\right)$ to be the the fundamental group of the simple complex of groups $C_{m}$. The geometric realization $|\mathcal{F}|$ is the derived complex of the Coxeter complex $\mathfrak{C}$ which is simply connected because $n \geq 4$. It follows that $\Gamma_{m}$ is in fact the direct limit

$$
\stackrel{\lim }{\longrightarrow}\left(\left(G_{\sigma, m}\right)_{\sigma \in F},\left(f_{a, m}\right)_{a \in E}\right) .
$$

There is a homomorphism $i_{m}: \Gamma_{m} \rightarrow S t_{n}(R)$ which restricts to an injection on each $G_{\sigma, m}$ into $S t_{n}(R)$ by the definition of $G_{\sigma, m}$ as a subgroup of $S t_{n}(R)$. 
Therefore the inclusion homomorphism $i_{\sigma, m}: G_{\sigma, m} \rightarrow \Gamma_{m}$ is injective and in particular the complex $\mathcal{C}_{m}$ is developable.

The following proposition allows us to identify each generator $x_{r}(a)$ of $S t_{n}(R)$ with its image in $\Gamma_{n}$ under $i_{\sigma, m}$ irrespective of the choice of flag $\sigma$.

Proposition 17. Let $g=x_{r}(a) \in S t_{n}(R), r \in \Sigma, a \in Y_{m}$ and $\sigma, \sigma^{\prime}$ be two flags in $F$ such that $r \in s(\sigma) \cap s\left(\sigma^{\prime}\right)$. Then $i_{\sigma, m}(g)=i_{\sigma^{\prime}, m}(g)$.

Proof. The collection of flags $\tau \in F$ such that $r \in s(\tau)$ is a convex subcomplex of $\mathfrak{C}$, in fact this is a hemisphere with boundary the great circle perpendicular to $r$. Therefore we may find a sequence $\sigma_{1}=\sigma, \sigma_{2}, \ldots, \sigma_{l}=\sigma^{\prime}$ of simplices of $\mathfrak{C}$ (i.e. flags in $F$ ) such that $r \in \cap_{i=1}^{l} s\left(\sigma_{i}\right)$ and $\sigma_{i} \cap \sigma_{i+1} \neq \emptyset$ for all $i=1, \ldots, k-1$. Therefore it is sufficient to prove the proposition in the case when the flag $\mu=\sigma \cap \sigma^{\prime}$ is non empty. Let $a=(\sigma, \mu), a^{\prime}=\left(\sigma^{\prime}, \mu\right)$ be the two edges of $\mathcal{F}$ and recall the inclusion maps $f_{a, m}: G_{\mu, m} \rightarrow G_{\sigma, m}$ and $f_{a^{\prime}, m}: G_{\mu, m} \rightarrow G_{\sigma^{\prime}, m}$ We have $f_{a, m}(g)=g=f_{a^{\prime}, m}(g)$ from the relations R3 in the definition of $\tilde{G}\left(\mathcal{C}_{m}\right)$ and the fact that $\mathcal{C}_{m}$ is simply connected. Therefore

$$
i_{\sigma, m}(g)=i_{\sigma, m} \circ f_{a, m}(g)=i_{\sigma^{\prime}, m} \circ f_{a^{\prime}, m}(g)=i_{\sigma^{\prime}, m}(g)
$$

as required.

From now on we shall identify the groups $G_{\sigma, m}$ with their images in $\Gamma_{m}$ under $i_{\sigma, m}$.

Note that the inclusions $Y_{m} \subset Y_{m+1}$ give rise to inclusions $G_{\sigma, m} \leq G_{\sigma, m+1}$ which, by the universal property of direct limits give rise to a homomorphism $F_{m}: \Gamma_{m} \rightarrow \Gamma_{m+1}$ such that $F_{m}$ restricted to $G_{\sigma, m}$ is the inclusion map of $G_{\sigma, m}$ into $G_{\sigma, m+1} \leq \Gamma_{m+1}$.

For every integer $m \geq 1$ let

$$
A_{m}=\left\langle G_{\sigma, 1} \mid \sigma \in F_{\max }\right\rangle \leq \Gamma_{m} .
$$

and note that $A_{m+1} \subseteq \operatorname{Im}\left(F_{m}\right)$.

Claim 18. $A_{m}=\Gamma_{m}$.

Proof of claim: Let $\sigma \in F_{\max }$. It is sufficient to show that for any integer $k \leq m$ the element $x_{r}(a) \in \Gamma_{m}$ with $a \in Y_{k}$ and $r \in \Sigma$ belongs to $A_{m}$. We prove this statement by induction on $k$, the case $k=1$ being clear from the definition of $A_{m}$.

Let us write $a=a_{1} a_{2}$ with $a_{1} \in Y_{k-1}$ and $a_{2} \in Y$. Using that the Weyl group $\operatorname{Sym}(n)$ acts transitively on the set $\Sigma$ of all roots we can find a maximal flag $\sigma^{\prime} \in F_{\max }$ and roots $r_{1}, r_{2} \in s\left(\sigma^{\prime}\right)$ such that $r=r_{1}+r_{2}$. Since $r \in s\left(\sigma^{\prime}\right)$ Proposition 17 gives $x_{r}(a) \in G_{\sigma^{\prime}, m}$. Now $x_{r_{1}}\left(a_{1}\right)$ and $x_{r_{2}}\left(a_{2}\right)$ belong to $A_{m}$ by the induction hypothesis and the identity $x_{r}(a)=\left[x_{r_{1}}\left(a_{1}\right), x_{r_{2}}\left(a_{2}\right)\right]$ in $G_{\sigma^{\prime}, m}$ gives that $x_{r}(a) \in A_{m}$. This completes the induction step and the claim follows. 
Since $\Gamma_{m+1}=A_{m+1} \subseteq F_{m}\left(\Gamma_{m}\right)$ we deduce that the maps $F_{m}: \Gamma_{m} \rightarrow$ $\Gamma_{m+1}$ are surjective for each $m$. The Steinberg group $S t_{n}(R)$ is a direct limit of of the directed system $\Gamma_{1} \rightarrow \Gamma_{2} \rightarrow \cdots$ because for every pair of roots $r_{1}, r_{2} \neq-r_{1}$ and every $a, b \in R$ there is some $k \in \mathbb{N}$ and a fundamental set of roots $s(\sigma)$ for some $\sigma \in F_{\max }$ such that $r_{1}, r_{2} \in s(\sigma)$ and $x_{r}(a), x_{r}(b) \in G_{\sigma, k}$. Now we use the following two results to deduce that $S t_{n}(R)=\Gamma_{m}$ for some integer $m$.

Theorem 19 ([16], Theorem 3). Suppose that $R$ is a unital finitely presented ring and $n \geq 4$. Then the Steinberg group $S t_{n}(R)$ is finitely presented.

Proposition 20. Let $B_{1} \rightarrow B_{2} \rightarrow \cdots$ be a directed system of surjective group homomorphisms $B_{i} \rightarrow B_{i+1}$ of groups $B_{i}$ such that $B_{1}$ is finitely generated while the direct limit $D=\lim _{\longrightarrow} B_{i}$ is finitely presented. Then the natural homomorphism $B_{m} \rightarrow D$ is an isomorphism for some integer $m \in \mathbb{N}$.

Proof. Let $N_{i}$ be the kernel of the composition map $B_{1} \rightarrow B_{i}$. We have $\{1\}=N_{1} \leq N_{2} \leq \cdots$ and $\cup_{i=1}^{\infty} N_{i}=H$ where $B_{1} / H=D$. Since $D$ is finitely presented, $H$ is generated by finitely many elements as a normal subgroup of $B_{1}$ and therefore $N_{m}=H$ for some $m$. In particular the homomorphism from $B_{m}=B_{1} / N_{m}$ to $B_{1} / H$ is an isomorphism.

Hence the natural homomorphism $i_{m}: \Gamma_{m} \rightarrow S t_{n}(R)$ is an isomorphism for some integer $m$.

For any flag $\sigma \in F$ we have $G_{\sigma, m}$ contains the nontrivial elements $x_{r}(a)$ for any $a \in Y$ and any root $r \in s(\sigma)$. In particular the centre of $G_{\sigma, m}$ contains an infinite cyclic subgroup $Z_{\sigma, m}<X_{r}$ for the highest root of $s(\sigma)$. Since by assumption $X_{r} \cap\left(\cap_{i} H_{i}\right)=\{1\}$ we deduce $\left[Z_{\sigma, m}: H_{i} \cap Z_{\sigma, m}\right] \rightarrow \infty$. By Proposition 7 with $G=G_{\sigma, m}$ and $N=Z_{\sigma, m}$ the chain $H_{i} \cap G_{\sigma, m}$ is economical in $G_{\sigma, m}$. Theorem 9 now follows from Theorem 8.

Proof of Corollary 10. Let $R=\mathbb{Z}\left[x_{1}, \ldots, x_{k}\right]$. Consider the homomorphism $\pi: S t_{n}(R) \rightarrow S L_{n}(R)$ which sends the generator $x_{r}(a) \in S t_{n}(R)$ with root $r=e_{i}-e_{j}$ to the elementary matrix $E_{i, j}(a)$. By Suslin's theorem [22] $S L_{n}(R)$ is generated by elementary matrices and therefore $\pi$ is surjective. The group ker $\pi$ is denoted by $K_{2}(n, R)$ and since $R$ has Krull dimension $k+1$ [14] gives that $K_{2}(n, R) \simeq K_{2}(R)$ and does not depend on $n$ when $n \geq k+4$.

In turn [21], p.122, Theorem 8 gives that $K_{2}(R)=K_{2}(\mathbb{Z})=\{ \pm 1\}$ and therefore $S L_{n}(R)$ is a factor group of $S t_{n}(R)$ by a central subgroup $\langle a\rangle$ of order 2. Let $H_{i}$ be a chain with trivial intersection in $S L_{n}(R)$ and consider the chain $\left(\pi^{-1}\left(H_{i}\right)\right)$ in $S t_{n}(R)$. Theorem 9 gives that $\left(\pi^{-1}\left(H_{i}\right)\right)$ is economical. Adding the single extra relation $a=1$ to the presentation of each $\pi^{-1}\left(H_{i}\right)$ now shows that $\left(H_{i}\right)$ is economical.

Proof of Proposition 11. The group $S L_{3}(R)$ has 6 root subgroups $E_{1}, \ldots$ $E_{6}$ isomorphic to $(R,+)$ which are in correspondence with the roots $r_{1}, \ldots r_{6}$ 
labelled clockwise on the realization of the root system $\Sigma=A_{2}$ as a regular hexagon. Let $D$ be the subgroup of $S L_{3}(R)$ of diagonal matrices. We have that $D \simeq R^{*} \times R^{*}$ is finitely generated by Dirichlet's unit theorem. For $i=1, \ldots, 6$ let $T_{i}=D \ltimes\left\langle E_{i} E_{i+1} E_{i+2}\right\rangle$. Each $T_{i}$ is isomorphic to the subgroup $T \leq S L_{3}(R)$ of upper triangular matrices. Let $\operatorname{Tr}_{3}(R)$ be the subgroup of upper triangular matrices in $G L_{3}(R)$ and let $Z$ be its subgroup of scalar matrices. Now $Z \simeq R^{*}$ is finitely generated while $\operatorname{Tr}_{3}(R)$ is finitely presented by [18, Proposition 11.2.8]. Since $T \cap Z=1$ we see that $T$ embeds as a subgroup of finite index in $\operatorname{Tr}_{3}(R) / Z$ and hence is finitely presented. Similarly we can see that each $T_{i} \cap T_{i+1}$ is finitely presented.

Let $\Gamma:=S t_{3}(R)$. Recall the definition of the subgroups $X_{r}<\Gamma(r \in \Sigma)$. Let $\pi: \Gamma \rightarrow S L_{3}(R)$ be the surjective homomorphism which sends $X_{r_{i}}$ to $E_{i}$. The kernel $K=\operatorname{ker} \pi=K_{2}(3, R) \simeq K_{2}(R)$ is a finite central subgroup of $\Gamma$ by [11]. Therefore the groups $\tilde{T}_{i}:=\pi^{-1}\left(T_{i}\right)$ and $\tilde{T}_{i} \cap \tilde{T}_{i+1}=\pi^{-1}\left(T_{i} \cap T_{i+1}\right)$ are finitely presented for $i=1, \ldots, 6$.

Let $L$ be the simply connected hexagonal 2-complex with six vertices $r_{i}$, six edges $\left(r_{i}, r_{i+1}\right)$ for $i=1, \ldots, 6$ and one disc $B$. Define $G_{r_{i}}=$

$\tilde{T}_{i}, G_{\left(r_{i}, r_{i+1}\right)}=\tilde{T}_{i} \cap \tilde{T}_{i+1}$ and $G_{B}=\pi^{-1}(D)$. Let $C$ be the resulting complex of groups. One checks that the canonical inclusion map $\tilde{T}_{i} \rightarrow \Gamma$ induces an isomorphism between $\tilde{G}(C)$ and $\Gamma$.

Let $\left(H_{i}\right)$ be a chain in $S L_{3}(R)$ with trivial intersection. Define $\tilde{H}_{i}=$ $\pi^{-1}\left(H_{i}\right)$. Since $K$ is finite it is sufficient to prove that $\left(\tilde{H}_{i}\right)$ is economical in $\Gamma$ and by Theorem 8 we just need to verify that $\left(G_{\sigma} \cap \tilde{H}_{i}\right)$ is economical in $G_{\sigma}$ for each cell $\sigma$ of $L$. Again using that $K=\operatorname{ker} \pi$ is finite it is sufficient to show that the chain $\left(\pi\left(G_{\sigma} \cap \tilde{H}_{i}\right)\right)=\left(H_{i} \cap \pi\left(G_{\sigma}\right)\right)$ is economical inside the finitely presented infinite soluble group $\pi\left(G_{\sigma}\right)<S L_{3}(R)$. Since $\cap_{i} H_{i}=\{1\}$ we are in position to apply Theorem 6.

\section{Artin Groups}

Let $K:=\left(V_{K}, E_{K}\right)$ be a graph such that every edge $e=(v, w)$ carries a weight $m_{e} \in\{2,3, \ldots\}$. The Coxeter matrix associated to $K$ is the symmetric matrix $M_{K}=\left(m_{(v, w)}\right)$ of size $|V|$. We define the Artin group $A_{K}$ and the Coxeter group $W_{K}$ associated to $K$ as follows:

$$
\begin{gathered}
A_{K}=\langle V \mid \underbrace{v w v w \ldots}_{m_{v, w}}=\underbrace{w, \ldots}_{m_{v, w} v v w v \ldots} \forall(v, w) \in E_{K}\rangle \\
W_{K}=\left\langle V \mid v^{2}=1 \forall v \in V ;(v w)^{m_{v, w}}=1 \forall(v, w) \in E_{K}\right\rangle
\end{gathered}
$$

It is clear that $A_{K}$ maps onto $W_{K}$. If $W_{K}$ is finite, then $A_{K}$ is called an Artin group of finite type. Artin groups of finite type were shown to have infinite cyclic centre by Bierskorn and Saito [6]. Cohen and Wales proved that they are linear [9]. Our Proposition 7 thus implies that an Artin group of finite type, by virtue of being a residually finite group with an economical normal subgroup, is economical. 
By a standard parabolic subgroup of $A_{K}$ or $W_{K}$, we mean any subgroup generated by a (possibly empty) subset of the standard generating set $V_{K}$. For $s \subseteq V_{K}$, let $K(s)$ denote the full subgraph of $K$ spanned by $s$. The inclusion of $s$ into $V$ induces a natural homomorphism $h_{s, V}: A_{K(s)} \rightarrow A_{K}$. A classical theorem by van der Lek [17] says that for any $s$, the map $h_{s, V}$ is an isomorphism onto its image.

The simplicial complex $L$, called the nerve of the Coxeter matrix $M_{K}$, is defined as follows: its vertex set is $V$ and a subset $s \subset V$ spans a simplex iff $W_{K(s)}$ is finite. Davis and Leary [10] computed the $l^{2}$-cohomology of $L$ and established that the $i$-th $\ell^{2}$-Betti number of $A_{K}$ is equal to the $(i-1)$-th ordinary reduced Betti number of $L$, provided the $K(\pi, 1)$ conjecture for Artin groups holds. In particular, if $L$ is connected and simply connected then both the first and second $\ell^{2}$-Betti numbers of $A_{K}$ should be zero.

Consider the partial order on the set $\mathcal{V}:=\left\{\emptyset \neq s \subseteq V:\left|W_{K(s)}\right|<\infty\right\}$ given by reverse inclusion that is $s_{1}<s_{2}$ whenever $s_{2} \subset s_{1}$. Let $P$ be the scwol associated to this partial order. For $s \in \mathcal{V}$ let $G_{s}=A_{K(s)}$. For an edge $a=\left(s_{1}, s_{2}\right)$ of $P$ with $s_{1}<s_{2}$ define the monomorphism $f_{a}: G_{s_{2}} \rightarrow G_{s_{1}}$ to be the inclusion map $h_{s_{2}, s_{1}}$.

Let $\mathcal{C}$ be the simple complex of groups $\left(P,\left(G_{s}\right), f_{a}\right)$. The geometric realization $|P|$ of $P$ is the derived complex of the nerve $L$, equal to the barycentric subdivision of $L$. Therefore $|P|$ is simply connected and $\tilde{G}(\mathcal{C})$ is the direct limit of the system $\left(\left(G_{s}\right)_{s \in \mathcal{V}},\left(f_{a}\right)\right)$ which is just $A_{K}$. We are now in a position to prove Theorem 12 .

Proof of Theorem 12. Suppose that $L$ is connected and simply connected so that $A_{K}$ is the fundamental group of the complex of groups over $\mathcal{C}$ as described above. Let $\left(H_{i}\right)$ be a chain of normal finite index subgroups of $A_{K}$ so that $\cap H_{i}$ is the radical $\mathfrak{R}\left(A_{K}\right)$. We will show that the chain $\left(H_{i}\right)$ is economical.

To apply Theorem 8, we need to check that for each simplex $\sigma \subset L$, the normal chain $\left(G_{\sigma} \cap H_{i}\right)$ is economical. We argue that $\left[Z_{\sigma}: H_{i} \cap Z_{\sigma}\right]$ is unbounded, where $Z_{\sigma}$ denotes the centre of $G_{\sigma}$. To do this we consider the homomorphism $p: A_{K} \rightarrow \mathbb{Z}$ that sends each $v \in V$ to the generator of $\mathbb{Z}$. Clearly, ker $p \geq \mathfrak{R}\left(A_{K}\right)$. Each $G_{\sigma}$ is of finite type and therefore by [6], $Z_{\sigma}$ is an infinite cyclic group generated by a suitable power of the Garside element $\prod_{v \in V(\sigma)} v$ (where $V(\sigma)$ is the set of vertices of the simplex $\sigma \subset L$ ). This implies that $p\left(Z_{\sigma}\right)$ is non-trivial and so, $Z_{\sigma} \cap \mathfrak{R}\left(A_{K}\right)=1$. We conclude that $\lim _{i \rightarrow \infty}\left[Z_{\sigma}: Z_{\sigma} \cap H_{i}\right]=\infty$ and by Proposition 7 , the chain $\left(G_{\sigma} \cap H_{i}\right)$ is economical, for each $\sigma \subset L$. We invoke Theorem 8 to conclude that the chain $\left(H_{i}\right)$ is economical in $A_{K}$.

\section{REFERENCES}

[1] M. Abert, D. Gaboriau, in preparation.

[2] M. Aschenbrenner, S. Friedl, H. Wilton, 3-manifold groups, arXiv/1205.0202v3. 
[3] M. Abert, N. Nikolov, Rank gradient, cost of groups and the rank versus Heegaard genus problem J. Eur. Math. Soc. Vol. 14, no. 5, 1657-1677, (2012).

[4] M. Abert, N. Nikolov, A. Jaikin-Zapirain, Rank gradient from combinatorial viewpoint, Groups Geom. Dynamics, Vol. 5, 213-230, (2011).

[5] B. Baumslag, S.J. Pride. Groups with two more generators than relators, J. London Math. Soc. (2) 17 (1978).

[6] E. Bierskorn, K. Saito, Artin-Gruppen und Coxeter-Gruppen, Invent. Math. 17, 245271, (1972).

[7] M. Bridson, A, Haeflliger. Metric spaces of non-postive curvature, Springer 1999.

[8] M. Bridson, D Koshloukova, Volume gradients and homology in towers of residually free groups, preprint http://arxiv.org/abs/1309.1877

[9] A. Cohen, D. Wales, Linearity of Artin Groups of finite type, Israel J. of Math., Vol. 131, 101-123 (2002).

[10] M. Davis, I. Leary, The $l^{2}$-cohomology of Artin groups, J. London Math. Soc. Vol. 68, 493-510, (2003).

[11] R.K. Dennis, M.R. Stein, The functor $K_{2}$ : A survey of computations and problems, Algebraic K-theory II Springer, Lecture Notes in Math 342 (1973), 243-280.

[12] C. Hog, M. Lustig and W. Metzler, Presentation classes, 3-manifolds and free products. In Geometry and topology, Lecture Notes in Math. 1167, 154-167, Springer, 1985.

[13] B. Eckmann, Introduction to $l_{2}$-methods in topology: reduced $l_{2}$-homology, harmonic chains and $l_{2}$-Betti numbers, Israel J. Math. 117 (2000), 183-219.

[14] W. van der Kallen, Injective stability for $K_{2}$. Algebraic K-theory (Proc. Conf., Northwestern Univ., Evanston, Ill., 1976), Lecture Notes in Math. 551, 77-154, Springer, 1976.

[15] A. Kar and N. Nikolov, Cost and Rank gradient of Artin groups and their relatives, to appear in Groups Geom. Dynamics 2014.

[16] S. Krstic, J. McCool, Presenting $G L_{n}(k\langle X\rangle)$, J. Pure and Applied Algebra 141 (1999), $175-183$

[17] H van der Lek, The Homotopy Type of Complex Hyperplane Complements, Ph.D. Thesis, University of Nijmegen (1983).

[18] J. Lennox, D. Robinson, The Theory of Infinite Soluble Groups, OUP, 2004.

[19] J. Lott, Deficiencies of lattice subgroups of Lie groups, Bull. London Math. Soc. 31 (1999) 191-195.

[20] J. Milnor, Introduction to algebraic K-theory Princeton University press, 1972.

[21] D. Quillen, Higher algebraic $K$-theory. I, Algebraic $K$-theory, I: Higher $K$-theories (Proc. Conf., Battelle Memorial Inst., Seattle, Wash., 1972), Springer, Berlin, 1973, pp. 85- 147. Lecture Notes in Math. 341.

[22] A. A. Suslin. On the structure of the special linear group over polynomial rings. Math. USSR Izv. 11, (1977) 221-238.

[23] B. Weiss, Monotileable amenable groups, Topology, ergodic theory, real algebraic geometry, Amer. Math. Soc. Transl. Ser. 2, 202, 257-262.

Mathematical Institute, University of Oxford, Andrew Wiles Building, OxFORD OX2 6GG, ENGLAND.

E-mail address: Aditi.Kar@maths.ox.ac.uk (Corresponding author)

E-mail address: Nikolay.Nikolov@maths.ox.ac.uk 